INRA Prod. Anim.,

2014, 27 (4), 303-316

\title{
Implications organisationnelles de la sélection génomique chez les bovins et ovins laitiers en France : analyses et accompagnement
}

\author{
J. LABATUT $T^{1,2,3,4}$, J.-M. ASTRUC ${ }^{5}$, F. BARILLET $T^{6,7,8,9}$, D. BOICHARD ${ }^{10,11}$, \\ V. DUCROCQ ${ }^{10,11}$, L. GRIFFON ${ }^{12}$, G. LAGRIFFOUL' \\ 1 INRA UMR1248 AGIR, F-31326 Castanet-Tolosan, France \\ 2 ENSAT UMR1248 AGIR, F-31326 Castanet-Tolosan, France \\ 3 Université de Toulouse INPT UMR1248 AGIR, F-31029 Toulouse, France \\ ${ }^{4}$ Université de Toulouse INPT, Ecole d'Ingénieurs de Purpan, UMR1248 AGIR, F-31076 Toulouse, France \\ 5 Institut de l'Elevage, BP42118, F-31321 Castanet-Tolosan, France \\ ${ }^{6}$ INRA, UMR1388 GenPhySE, F-31326 Castanet-Tolosan, France \\ 7 Université de Toulouse INPT ENSAT, UMR1388 GenPhySE, F-31326 Castanet-Tolosan, France \\ 8 Université de Toulouse INPT ENVT, UMR1388 GenPhySE, F-31076 Toulouse, France \\ ${ }^{9}$ Université de Toulouse INPT, Ecole d'Ingénieurs de Purpan, UMR1388 GenPhySE, F-31076 Toulouse, France \\ 10 INRA, UMR1313 GABI, F-78352 Jouy-en-Josas, France \\ 11 AgroParisTech, UMR1313 GABI, 16 rue Claude Bernard, F-75321 Paris, France \\ 12 Institut de l'élevage, 149 rue de Bercy, F-75012 Paris, France
} Courriel :julie.labatut@toulouse.inra.fr

Le déploiement de la sélection génomique chez les ruminants s'accompagne de changements organisationnels importants dans les activités de recherche et les activités de sélection. Cet article présente une synthèse des premiers travaux visant à analyser et accompagner ces changements dans les filières laitières bovines et ovines.

Les méthodes et les pratiques de sélection connaissent depuis quelques années de profondes mutations, notamment avec le développement de la sélection génomique. Cette nouvelle méthode, mise en œuvre en 2009 pour les trois principales races bovines laitières françaises, est présentée comme une révolution à la fois scientifique (Peerbaye 2004) et technique (Bidanel et al 2008, Guillaume et al 2011, Institut de l'Elevage et INRA 2011, Robert-Granié et al 2011, Boichard et al 2012), équivalente au développement de l'insémination animale. En effet, d'un point de vue scientifique, la génomique est considérée comme une rupture épistémologique et comme un espace cognitif nouveau (Peerbaye 2004). Comme l'indique cet auteur, une telle rupture permet de construire et d'explorer des « espaces de problèmes qui étaient auparavant trop grands pour l'être ", notamment au travers de masses colossales de données, mais aussi de concevoir de nouveaux espaces de recherches et d'applications. La génomique conduirait également à réviser la place respective des expériences, des hypothèses et des données dans la production de connaissances scientifiques (Peerbaye 2004). Du point de vue de son application en sélection animale, la génomique est vue comme une ouverture du champ des possibles, permettant d'évaluer le potentiel génétique d'un animal sans avoir à le tester sur sa descendance.

Mais la sélection génomique n'a pas que des implications techniques fortes, elle s'accompagne également de changements organisationnels importants dans les filières de la sélection animale, d'une part en amont pour le développement de cette méthode et, d'autre part, en aval pour gérer les modifications induites par sa mise en place. Ceux-ci s'inscrivent en France dans une évolution politique du secteur de la génétique animale au travers de la réforme de la Loi sur l'Elevage dans le cadre de la Loi d'Orientation Agricole de 2006. Ces changements ont été relativement peu analysés jusqu'à présent, notamment du fait de leur caractère récent et pour certains d'entre eux, transitoires. Pourtant, il est important pour les différents acteurs de la sélection animale, qu'il s'agisse des organismes de recherche et de développement comme des entreprises et organismes de sélection, de repérer et d'analyser ces changements pour les accompagner au mieux (Verrier et Saint-Dizier 2011), tout en maintenant les capacités collectives actuelles et futures de sélection des populations animales. Ainsi, si ces changements sont déjà à l'œuvre dans quatre races bovines laitières présentes en France (Montbéliarde, Normande, Holstein et Brune), les acteurs d'autres espèces (ovins laitiers notamment mais aussi caprins) et d'autres races bovines (races allaitantes et races à plus faibles effectifs) s'interrogent sur la manière de saisir et développer les opportunités techniques de la sélection génomique tout en anticipant ces changements.

Plusieurs travaux et projets ont ainsi été conduits depuis 2010 visant à la fois à explorer, analyser et accompagner ces changements dans les filières bovines laitières et allaitantes, et ovines laitières. Après une première phase exploratoire (2010) ayant permis de réaliser des enquêtes auprès des acteurs nationaux principaux de la sélection (INRA, Institut de l'Elevage, UNCEIA ${ }^{1}$, Races de France)

${ }^{1}$ Union Nationale des Coopératives d'Insémination Artificielle. 
deux projets interdisciplinaires (entre science de gestion et génétique) ont été conduits et valorisés (Astruc et al 2012, Labatut 2013, Labatut et al 2013a, Labatut et al 2013b) :

- Le projet STRING (Stratégies d'Acteurs face à l'Innovation Génomique) financé par le département $\mathrm{SAD}^{2}$ de l'INRA puis par Apis-gène et $\mathrm{FGE}^{3}$, analysait ces évolutions dans le cas des bovins. Ce projet portait à la fois sur des races pour lesquelles la sélection génomique était déjà à l'œuvre (Montbéliarde, Limousine, Brune) et d'autres pour lesquelles elle est au stade de programmes de recherche (Tarentaise, Aubrac).

- Le projet COSEGOV (refondation des COntrats de SElection et d'utilisation en OVin en lien avec l'émergence de la Génomique), financé par FGE, se centrait sur les races ovines laitières des trois principaux bassins français (Lacaune, Manech Tête Rousse, Manech Tête Noire, Basco-Béarnaise et race Corse). De façon prospective (la sélection génomique était au stade de recherche et de développement), ce projet visait à accompagner les acteurs des schémas de sélection en ovins laitiers dans leur réflexion sur l'évolution du fonctionnement organisationnel des schémas et l'adaptation des contrats de sélection dans le cas du développement de la sélection génomique.

Ces deux projets associaient à chaque fois le département SAD et les départements Génétique Animale de l'INRA et de l'Institut de l'Elevage. Deux méthodes ont principalement été mises en œuvre dans ces travaux. Dans le cadre du projet STRING, nous avons travaillé principalement par entretiens (avec une diversité d'acteurs, éleveurs, responsables d'organismes et d'entreprises de sélection, acteurs de la recherche et du développement...), observations (lors de réunions professionnelles, de colloques scientifiques et professionnels...) et analyse de documents professionnels et scientifiques. Dans le cadre du projet COSEGOV, la méthode utilisée a reposé sur l'organisation d'ateliers collectifs de réflexion, conjugués à des entretiens individuels. Ces ateliers réunissaient, par Entreprise de Sélection puis par Organisme de Sélection, les responsables techniques et les éleveurs impliqués dans les conseils d'administration.

Nous rendons ici compte des premiers résultats issus de ces deux projets. Dans un premier temps, nous rappellerons les principes organisationnels sur lesquels ont été fondées les activités de sélection génétique des ruminants jusqu'à la révolution génomique et la réforme de la Loi sur l'Elevage. Dans un deuxième temps, nous présentons les résultats obtenus dans le cadre des enquêtes auprès des races bovines, en identifiant quatre dimensions dans les changements observés. Enfin, nous détaillons les pistes identifiées lors des ateliers de réflexion menés avec les acteurs des filières ovines laitières.

\section{1 / Sélectionner des ruminants}

\section{1 / Constitution d'un régime de sélection coopératif et public}

Afin de comprendre les enjeux organisationnels actuels autour de la sélection génomique des ruminants, il est important de rappeler les principes historiques d'organisation des acteurs et des activités sur lesquels cette sélection a été réalisée. En effet, les activités de sélection des ruminants comportent des spécificités organisationnelles par rapport à celles de la sélection des volailles ou encore de la sélection végétale, qui sont principalement caractérisées par i) une forte intégration verticale, ii) la sélection par lignées et la production d'individus croisés et iii) des droits de propriété privée sur ces ressources (Larivière et Leroy 2008, Bonneuil et Thomas 2009). D'une part, pour des raisons biologiques (le nombre d'animaux que l'on peut réunir par hectare ou la faible capacité de multiplication des femelles reproductrices, par exemple) et de structure des exploitations françaises (de bien trop petite taille pour constituer isolément des noyaux de sélection), sélectionner les ruminants a impliqué d'organiser les fermes privées en un réseau d'élevages à la fois producteurs et utilisateurs du progrès génétique. D'autre part, la Loi sur l'Elevage de 1966 a défini les races comme la propriété collective d'une communauté d'éleveurs qui s'accordent sur la définition de ses caractéristiques. Les races animales correspondent donc à ce que l'on peut appeler des « communs » (Ostrom 1990, Labatut et al 2013a) : ces ressources ne font pas l'objet de droits de propriété privée, elles sont en libre accès, mais il peut y avoir « rivalité » sur la ressource. Par exemple, une trop forte utilisation d'un faible nombre de reproducteurs peut conduire à une augmentation de la consanguinité et une mise en danger de la race pour le futur. A l'inverse, la trop faible utilisation d'une race peut conduire à sa disparition. Mais il est important de noter que ce qui est commun dans la sélection des races animales, ce sont bien sûr des ressources biologiques, mais également des ressources informationnelles, qu'il s'agisse de don- nées généalogiques ou génétiques (Labatut et al 2013a). Ainsi depuis plusieurs siècles, les activités de sélection se sont accompagnées de la création de dispositifs de gestion de ces informations (par exemple, les Livres Généalogiques). Ce caractère « commun » est une construction à la fois sociale et technique. Ainsi, contrairement aux variétés, la sélection des races de ruminants a reposé sur la construction de dispositifs collectifs basés sur la coopération entre une diversité d'acteurs. Historiquement, l'Etat et les scientifiques ont considéré que c'était au moyen d'un encadrement fort des activités de sélection et d'une politique substantive pour générer et traiter la grande quantité d'informations génétiques nécessaires à la sélection des animaux au travers du « testage sur descendance ", ou des "stations de contrôle de performances » que le niveau génétique du cheptel ruminant français pourrait s'améliorer de manière efficace afin de pouvoir atteindre les niveaux de pays tels que les Pays-Bas ou les EtatsUnis, pour les bovins laitiers (Vissac 2002, Labatut 2009, Labatut et al 2011).

Pour favoriser une sélection efficace du cheptel français, l'Etat a donc à la fois organisé la division du travail de sélection, centralisé la production et la gestion des données génétiques, et encadré les marchés du progrès génétique. Ainsi jusqu'au début des années 2000, l'Etat a financé une part importante des activités de sélection des ruminants, et organisé ces activités au travers de la CNAG (Commission Nationale d'Amélioration Génétique). C'est à l'INRA et à ce qui est devenu l'Institut de l'Elevage que l'Etat a confié la gestion des systèmes nationaux d'information génétique, dans lesquels ces informations sont « publiques » au sens où elles sont partagées par l'ensemble des acteurs de la sélection. La fonction régalienne de calcul des estimations de valeur génétique des animaux que l'on appelle index a été confiée à l'INRA. Leur diffusion a quant à elle été confiée à l'Institut de l'Elevage. La production des données de performance nécessaires au calcul des valeurs génétiques est assurée par les organismes de Contrôle de Performance qui bénéficiaient jusqu'en 2015 d'une protection contre la concurrence, pour assurer la couverture territoriale. Les orientations de sélection des races, ainsi que leur promotion et la tenue des Livres Généalogiques, ont été dévolues aux UPRA (Unions pour la sélection et la Promotion des Races, devenues Organismes de Sélection à partir de 2006), réunissant l'ensemble des usagers

\footnotetext{
2 Sciences pour l'Action et le Développement.

3 France Génétique Elevage.
} 
de la race, et reconnues comme des «Parlements » définissant la gestion des races en tant que Communs. L'Etat a organisé le marché de la semence en définissant un monopole de zone pour les coopératives d'Insémination Animale (IA), dans le but de limiter la " guerre des prix » et d'assurer un prix et un accès équitables à la génétique via l'IA à tous les éleveurs français. Ce monopole territorial et l'orientation des races prises à l'échelle des UPRA entraînaient une offre relativement équivalente de la part de l'ensemble des coopératives de sélection (peu de stratégies différenciées). Ce dispositif collectif et public permettait donc de produire et gérer à la fois les ressources biologiques et les ressources informationnelles nécessaires à la sélection. Il s'est révélé d'une grande efficacité, notamment grâce à la mutualisation des efforts de recherche et a permis à la France de rattraper ses concurrents internationaux en termes de niveau génétique.

\section{2 / Des bouleversements tech- niques et politiques}

Différentes évolutions à la fois techniques et politiques ont amené à reconfigurer ce régime de sélection (Labatut et al 2013a). De 2001 à 2008, des collaborations entre l'INRA et l'UNCEIA (Union Nationale des Coopératives d'Insémination Artificielle) ont permis le développement de la Sélection Assistée par Marqueurs (SAM), nécessitant auparavant une détection de QTL ( $q$ quantitative trait loci $»$ : région du génome à caractère d'intérêt quantitatif) ${ }^{4}$. Des structures support de cette nouvelle forme de partenariat public-privé sont apparues : le GIS Agenae réunissant au départ l'INRA et le CIRAD d'un côté et les filières aquacoles et bovines de l'autre, et visant à orienter les recherches autour de la génomique ; Apis-gène, société par actions simplifiée constituée par l'UNCEIA, le CNIEL, INTERBEV, la CNE et l'Institut de l'Elevage, finançant une partie de ces recherches. A partir de 2003-2004, puis avec le séquençage du génome bovin, les travaux sur les SNP à haut débit débutent (technologies de génotypage à haut débit par puces à SNP, "Single Nucleotide Polymorphism »). Une première SAM sur SNP à haut débit, dite SAM2, a été mise en œuvre dès l'automne 2008, donnant lieu à des index officiels mâles en juin 2009 (Institut de l'Elevage et INRA 2011).

Parallèlement à ces évolutions scientifiques et organisationnelles, le système de sélection français, et notamment le monopole de zone des coopératives d'insémination, est soumis aux coups de boutoirs de concurrents nationaux ou internationaux qui souhaitent développer leur activité d'insémination ou de vente de semence sur le marché français, sans passer par les coopératives d'insémination. Ainsi le Conseil de la concurrence français condamnera la filière à une amende pour entrave à la libre concurrence. Les acteurs de la sélection génétique s'investissent alors de manière proactive à la refondation de l'organisation du secteur telle que définie dans la Loi sur l'Elevage de 1966, au travers de la réforme de la Loi d'Orientation Agricole de 2006, modifiant radicalement l'organisation de la sélection (Verrier et al 2010). Cette réforme répond aussi à la volonté de l'Etat de réduire sa participation financière aux activités de sélection. Dans cette nouvelle Loi, le monopole de zone est supprimé et remplacé pour les zones les plus difficiles par un
Service Universel de l'Insémination Artificielle (SUIA). Le marché des biens et des services génétiques est donc ouvert sur les territoires français. Les centres de production de semence deviennent des Entreprises de Sélection (ES), et les UPRA des Organismes de Sélection (OS), au sein desquels la place des filières est renforcée (Verrier et al 2010). Dans ce milieu plus concurrentiel, l'Etat réaffirme le rôle de l'INRA comme seul organisme agréé à calculer des index « officiels». L'Etat délègue une partie de la gouvernance du dispositif génétique français à la profession avec la création de FGE, qui organise le périmètre des activités mutualisées et participe davantage au financement du dispositif.

La première évaluation utilisant les informations des puces pangénomiques dites « 50k » (permettant de typer 50000 marqueurs de type SNP répartis sur tout le génome) est réalisée en octobre 2008, mais ces index génomiques restent d'un usage « privé » pour les ES. C'est en 2009 que les travaux conduits par la recherche et les acteurs de la sélection sont officiellement diffusés dans les trois principales races bovines laitières françaises : la Holstein, la Normande et la Montbéliarde, qui reçoivent leurs premiers index génomiques « officiels » et peuvent alors diffuser la semence de taureaux sur la base de leurs index génomiques. Cette nouvelle méthode de sélection permet alors d'obtenir sans testage sur descendance des index (dits " génomiques ») ayant une fiabilité suffisante pour sélectionner les reproducteurs pour la vente de doses de semence (figure 1).

Quels sont les changements organisationnels et institutionnels dans les activités de sélection des bovins qui ont

Figure 1. Déroulement de l'évaluation génétique des taureaux, avant et après la sélection génomique (inspiré de Rousseau 2012).

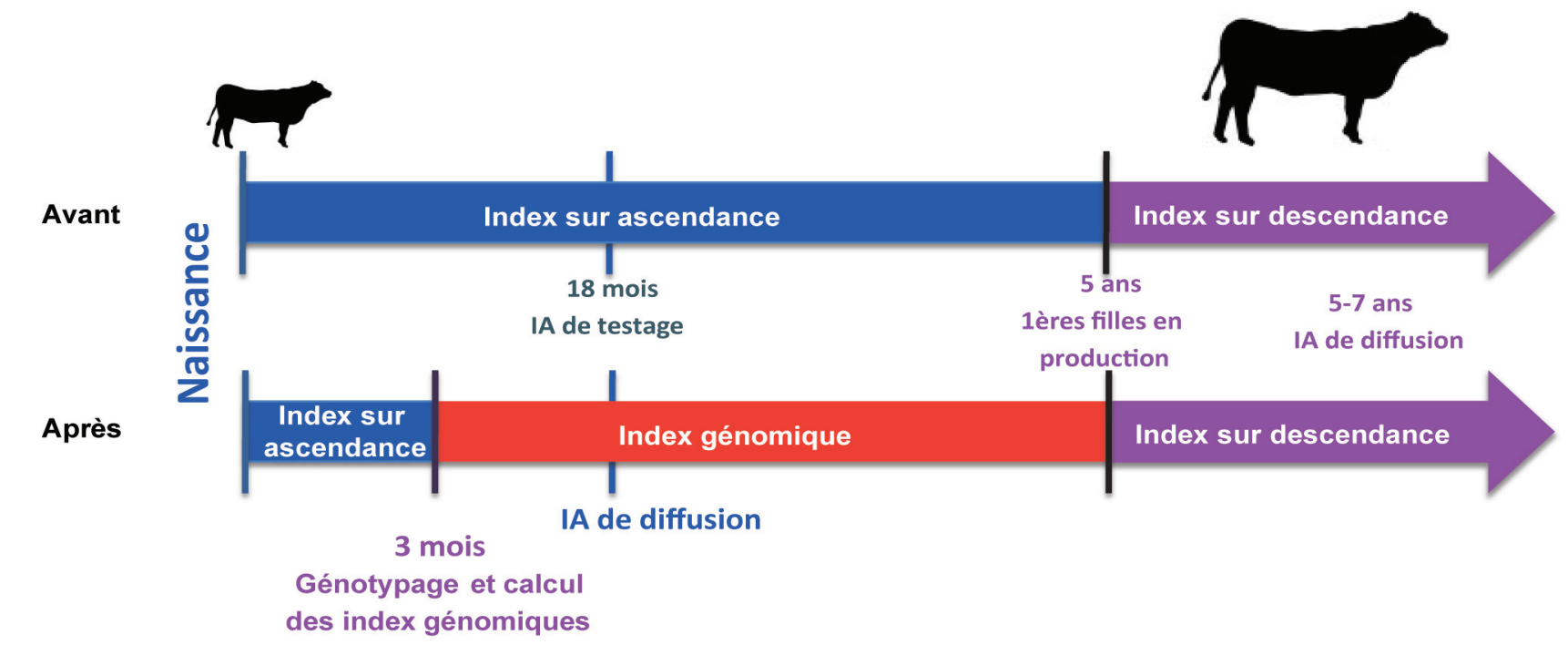

${ }^{4}$ Programme de recherche privé initié dès le milieu des années 1990 pour la recherche de QTL. 
accompagné cette innovation dans un contexte de réforme politique ? L'objet de la partie suivante est de fournir quelques éléments de réponse à cette question.

\section{2 / Implications organisation- nelles dans la filière bovine : quatre dimensions soulevées}

Les changements au sein des activités de sélection liés au développement de la sélection génomique et de la réforme de la Loi sur l'Elevage sont fortement interconnectés et traversent les différentes fonctions organisationnelles impliquées (des activités de production génétique, de calcul des index, de contrôle de performances...). Pour les expliciter, nous proposons ici un découpage en quatre grands axes de changements. Le premier concerne les activités de recherche et de développement autour de la sélection génomique. Le deuxième est celui des changements observés au niveau des entreprises de sélection. Le troisième est celui des rapports entre acteurs de la sélection et les éleveurs. Le quatrième concerne la gouvernance des races. Pour chacun de ces axes, nous évoquons les opportunités et les risques que ces changements peuvent amener. Nous observons ces changements dans l'action. Certains sont donc sans doute transitoires, d'autres s'institutionnaliseront peut être, ce que la continuité de ces travaux permettra d'observer.

\section{1 / Reconfiguration des rapports entre la recherche publique et les acteurs de la sélection}

Comme évoqué dans le point 1.2 , les activités de recherche et de développement sur les outils de la sélection génomique ont reposé sur la mise en place de nouvelles formes de coopération entre acteurs. Ainsi huit entreprises de sélection, ${ }^{5}$, engagées dans la sélection des races Holstein, Montbéliarde et Normande, ont formé un consortium et conclu un contrat exclusif avec l'INRA et le GIE LABOGENA $^{6}$. Cette collaboration spécifique, initiée en 2001, est une nouvelle forme de coopération entre la recherche publique et les acteurs de la sélection. Différents projets de recherche ont ainsi été cofinancés entre l'Agence Nationale de la Recherche, l'INRA et Apis-gène (par exemple les programmes Cartofine, 20062008, et AMASGEN, 2010-2012). Ce partenariat s'est traduit par des changements organisationnels concrets. Ainsi, tandis qu'avant les années 2000 les seules recherches menées à l'UNCEIA étaient tournées vers la reproduction (à l'Ecole Vétérinaire de Maisons-Alfort), l'UNCEIA et l'INRA ont créé un poste d'interface entre les deux organisations, poste d'ingénieur embauché par l'UNCEIA, mais en étroite collaboration avec l'INRA pour le développement de la SAM.

Ces relations de coopération ont également accompagné la formation d'un nouveau niveau de production et de gestion de données génétiques : les populations de référence. Une population de référence est une population d'animaux génotypés avec des informations sur leurs performances. C'est à partir de cette population que l'on peut mettre en relation phénotypes et génotypes, permettant ensuite de prédire les valeurs génétiques de jeunes animaux à travers des index génomiques sans avoir connaissance de leurs performances réelles ou de celles de leurs descendants. Comme l'indique l'UNCEIA dans l'un de ses communiqués de presse, « ces recherches ont bénéficié du système mutualisé français permettant d'accéder dans les meilleures conditions aux animaux et à leurs informations ». Cependant, c'est la première fois (au moins à cette échelle) que le partenariat entre la recherche publique et les acteurs de la sélection aboutit à la construction d'outils privés réservés aux entreprises de sélection membres du consortium ayant investi dans ces recherches. Trois générations d'outils ont ainsi vu le jour : la sélection assistée par marqueurs microsatellites de première génération, dite SAM1, de 2001 à 2007 ; son extension à un plus grand nombre de QTL suivis par des marqueurs SNP, ou SAM2, en 2008-2009; une généralisation à un grand nombre de QTL couvrant tout le génome, dite Sélection Assistée par Marqueurs Généralisée (SAMG) ou plus généralement Sélection Génomique (SG) à partir de 2010. Par la suite, pour valoriser et ouvrir cet outil privé, les entreprises de sélection membres du consortium ont créé en 2010 la société Valogène, ayant jusqu'en 2015 l'exclusivité de la licence d'exploitation du procédé d'évaluation génomique des bovins laitiers et de la fourniture de ce service aux éleveurs ou organismes souhaitant y avoir accès en race Holstein, Normande et Montbéliarde. L'INRA reste propriétaire du procédé et responsable de sa mise en œuvre. Cet accord inédit de valorisation devait assurer le retour sur investissement des entreprises de sélection et la « mobilisa- tion continue des efforts de recherche et d'innovation $\gg{ }^{7}$ tout en ouvrant l'utilisation à tous. Valogène permet également de mutualiser les commandes de puces à SNP pour obtenir des tarifs raisonnables pour la filière bovine, que ce soit pour les recherches ou pour l'application et la réalisation de la sélection génomique.

Ces nouvelles formes de partenariats public-privé et de mutualisation des investissements de recherche s'accompagnent en parallèle d'un accroissement des dynamiques privées et individuelles de recherche, et de l'éclatement de ces compétences. Comme indiqué dès 2010 par l'UNCEIA dans son rapport d'orientation, les entreprises de sélection cherchent à se différencier de leurs concurrents. L'UNCEIA rappelait l'importance d'articuler les recherches collectives et ces dynamiques de différenciations. Comme le souligne le responsable de l'UNCEIA dans le rapport d'orientation 2010 : «Certes, je comprends le souci des uns et des autres de se différencier de leurs concurrents. Cela est possible mais impose que l'on sépare bien les travaux de fond qui permettront la mise au point de techniques innovantes des travaux d'application à tel ou tel caractère pour créer la différence entre entreprises. Parce qu'ils sont onéreux, qu'ils s'inscrivent dans la durée et que leurs résultats ouvrent sur diverses applications, les premiers sont à conduire collectivement alors que les seconds relèvent de la stratégie propre à chaque entreprise ".

Les investissements individuels des entreprises et organismes de sélection dans des activités de recherche et de développement visant la différenciation vis-à-vis des concurrents prennent plusieurs formes. On observe le développement, au sein de certaines entreprises de sélection, de compétences de recherche propres, ainsi que de partenariats avec de nouveaux types d'acteurs (privés ou publics) qui n'étaient auparavant pas impliqués dans des activités de recherche en génétique animale en France. Une entreprise de sélection a par exemple créé une plateforme génomique interne, en nouant des relations avec une fondation de recherche privée et a récemment proposé la diffusion d'indicateurs non officiels sur de nouveaux caractères autour de la santé du pied. Un organisme de sélection a créé, en partenariat avec un laboratoire universitaire, une start-up de biotechnologie proposant ses propres outils d'évaluation génomique ${ }^{8}$. On observe également

\footnotetext{
5 Jura Bétail et Umotest en race Montbéliarde, GNA en race Normande, UNECO, GENOE, Midatest, URCEO et GDH en race Holstein.

6 Laboratoire d'Analyses Génétiques pour les Espèces Animales.

7 Dossier de presse du 9 septembre 2010, « La sélection génomique, une révolution pour la sélection des vaches laitières ». Accord de partenariat entre l'INRA, l'UNCEIA et Apis-gène, 8 pages.

8 Ingenomix.
} 
le développement de relations directes entre des entreprises et des laboratoires français ou étrangers pour certains programmes de recherche, sans passer par la mutualisation des demandes et des programmes que le Dispositif Génétique Français avait organisé en confiant ce travail à l'INRA. L'INRA n'est plus considéré comme le seul partenaire de recherche.

Ces démarches peuvent avoir différents objectifs : le développement d'outils d'évaluation génomique pour la vente de ce service (parfois pour des races pour lesquelles les outils génomiques n'ont pas encore été développés par les consortiums nationaux), et/ou le développement de recherches sur des caractères spécifiques innovants pour lesquels les entreprises développeraient des activités de phénotypage puis des gammes de taureaux différenciées de leurs concurrents. Le développement de ces recherches sur des données phénotypiques privées, des caractères spécifiques, est en effet vu comme une opportunité d'avantage compétitif dans un contexte de plus en plus concurrentiel. Dans ce contexte, l'organisation historique du Système National d'Information Génétique (SNIG) bovin, c'est-à-dire avec accès partagé aux données et mutualisation totale de celles-ci, est questionnée face à cette augmentation de la concurrence sur les données génétiques. La segmentation du statut des données (selon qu'elles sont publiques ou à accès plus ou moins limité, voire privatives), se traduit par des droits restreints d'accès aux données, voire à la remise en cause de la gestion de certaines données dans la base nationale gérée par l'INRA.

Ces nouvelles formes de partenariat et le développement des capacités individuelles de recherche, de développement et d'application de l'évaluation génomique comportent à la fois des risques et des opportunités. L'une des opportunités, en diversifiant et démultipliant les démarches de recherche, et en différenciant l'offre, est de favoriser l'innovation pour mieux répondre à la diversité des attentes des utilisateurs des races, en amont (éleveurs) comme en aval (filières de valorisation). Cependant, plusieurs risques peuvent être identifiés. D'une part, la forme de partenariat qui a permis aux trois races Holstein, Montbéliarde et Normande de développer la sélection génomique n'est pas aussi facilement reproductible pour d'autres races, pour lesquelles les capacités d'investissement et le poids de l'insémination artificielle par rapport à la monte naturelle seraient moindres, ainsi que la quantité de données issues du contrôle de performance. Par exemple, l'un des programmes de recherche en cours sur les perspectives de la génomique combinant les informa- tions de plusieurs races (GEMBAL) dont l'enjeu est pourtant considérable pour un grand nombre de races, a rencontré de fortes difficultés à reproduire ce type de partenariat du fait du nombre d'acteurs impliqués qui est beaucoup plus important, de rapports de force et de questions de pouvoir entre ces acteurs (notamment le rapport entre la capacité d'investissement financier et celle de l'apport en matériel biologique). Par ailleurs, les opérateurs sont confrontés au risque d'une perte d'efficacité des activités de recherche. En effet, l'éclatement des capacités de recherche mutualisées, qui ont fait le socle commun du dispositif génétique français au cours des décennies passées, amène différents opérateurs à investir dans des recherches similaires en parallèle, tout en ayant une moindre capacité d'investissement que dans le cas de la mutualisation. Cela pourrait entraîner le risque d'une hétérogénéité de qualité des évaluations pour une dénomination identique (plusieurs niveaux de qualité des index en fonction des biais potentiels et de leur précision) et poserait la question de la transparence des méthodes utilisées (s'il n'y a pas de publication officielle par exemple) ainsi que de leur objectivité (dans la mesure où les vendeurs de semence seraient aussi les évaluateurs de ces mêmes semences), ce qui était l'une des motivations de l'Etat à confier l'évaluation à un tiers n'ayant aucun intérêt dans les programmes de sélection. Certaines de ces recherches seraient menées plus efficacement de manière collective. Un risque supplémentaire est que la privatisation des recherches conduise à réduire d'autant les investissements tournés vers les races et espèces de moindre importance en termes d'effectif ou de rentabilité économique pour les entreprises de sélection.

\section{2 / Les relations entre les opé- rateurs de la sélection et de la production de données}

Dans cette partie nous abordons dans un premier temps les changements entre les opérateurs de la sélection proprement dite (ES et Entreprises de Mise en Place - EMP), puis dans un deuxième temps nous évoquons l'évolution des rapports entre ces opérateurs et ceux impliqués dans la production de données de phénotypage, qui reste à approfondir face à l'ouverture à la concurrence des services de contrôle de performance en 2015.

Ainsi, l'un des changements les plus importants dans l'organisation de la sélection des bovins a été le regroupement de nombreuses ES et EMP françaises. Si l'origine de ce mouvement est antérieure à l'arrivée de la sélection génomique, il s'est accéléré ces dernières années, dès la réforme du Dispositif Génétique Français en 2006 dans un premier temps, puis avec le développement de la sélection génomique. Ce mouvement a pris diverses formes. Pour les entreprises de mise en place, l'objectif a été avant tout de faire face à la suppression du monopole de zone et de se préparer à affronter une concurrence accrue. Pour les entreprises de sélection, les objectifs sont de favoriser les économies d'échelle, d'assurer l'accès à de nouvelles technologies, d'accroître les parts de marché. Ainsi, le groupe Evolution, dans le grand Ouest, illustre ce mouvement, avec l'union de GENOE et de l'URCEO en 2008, formant Créavia, puis l'union de Créavia avec Amélys en 2013, formant le groupe Evolution, deuxième groupe européen de sélection animale aujourd'hui. Accélérer la progression du groupe dans les nouvelles technologies, notamment la génomique, mais également le sexage de la semence et les services à l'éleveur, est clairement mis en avant comme l'une des justifications de ce regroupement.

Si dans le cas d'Evolution, le regroupement va jusqu'à la fusion en une seule organisation, d'autres formes de regroupements peuvent être observées. Ainsi certaines entreprises de sélection ont regroupé une part de leurs ressources, mais ont gardé des entités séparées et une partie de leurs activités indépendantes. C'est le cas de Midatest, entreprise de sélection du Sud-Ouest, qui a mutualisé son programme Holstein avec Evolution : «En race Holstein, nous avons encore un programme qui est conduit avec Evolution : nous représentons $10 \%$ des volumes et avons droit à $10 \%$ des taureaux, contre 90\% pour Evolution. L'enjeu principal de la génomique est la capacité à recruter des candidats de façon très large, puis de les trier efficacement. Aujourd'hui, le fait d'être adossés à ces partenaires-là fait que nous avons un nombre de taureaux disponibles sans commune mesure à ce que Midatest aurait seul. C'est le principal avantage" (responsable Midatest). C'est aussi pour pouvoir bénéficier de la technologie de sexage de la semence en Holstein que Midatest a mutualisé son programme Holstein.

Mais ce sont aussi les relations entre entreprises de sélection et entreprises de mise en place de la semence qui ont évolué. En effet, historiquement, certaines entreprises de sélection ont au départ été créées à travers la mutualisation de l'activité de production de semences par les coopératives d'insémination : «dans les années 1970, on a créé Midatest en partenariat avec d'autres coopératives. A l'époque, chaque coopérative avait son programme et les doses et les taureaux étaient mis en commun dans Midatest ». Aujourd'hui, certains acteurs considèrent que le rapport entre entreprises de sélection et coopératives d'inséminations s'est 
inversé, comme l'exprime l'un des directeurs d'ES rencontrés : «Les coopératives avaient la légitimité du terrain et considéraient que l'Entreprise de Sélection représentait les services communs, le " back office ». [...] Aujourd'hui [...] avec la génomique [...] le back office prend le leadership » (entretien directeur d'entreprise de sélection, 2011).

En revanche, dans d'autres cas, les relations entre certaines entreprises de sélection ont plutôt évolué vers une remise en question de rapports de coopération historiques et une augmentation de la compétition, notamment concernant les investissements de recherche dans le phénotypage de nouveaux caractères. Ainsi, deux entreprises importantes qui collaboraient jusqu'alors dans des projets de recherche en partenariat avec les instituts de recherche et développement (INRA et Institut de l'Elevage) autour de nouveaux critères de sélection, ont récemment développé des stratégies divergentes. L'une a continué sa collaboration historique avec ces instituts, tandis que l'autre n'a pas souhaité mutualiser ces recherches, pariant sur une stratégie de différenciation pour faire face à l'augmentation de la concurrence nationale et internationale. Ainsi, si la logique de « coopétition » (coopération entre concurrents) a été largement mise en avant au début de la sélection génomique (comme par exemple lors de l'Assemblée Générale de l'UNCEIA en février 2011), cela n'a clairement pas suffi à assurer le maintien du travail historique de mutualisation des activités. Les risques sont alors, pour les collectifs concernés (l'ensemble des entreprises, les éleveurs et leurs races), d'investir deux fois dans les mêmes recherches avec des moyens moindres (et donc potentiellement des résultats moins bons), ou de voir la sélection française prendre du retard sur certains caractères innovants (sanitaire, par exemple) pour les races pour lesquelles la coopération entre l'ensemble des partenaires se délite.

On observe donc à la fois des opportunités d'investissements mutualisés, et des acteurs privilégiant des stratégies individuelles pour tenter de développer un éventuel avantage compétitif et faire face à la menace de la compétition, révélant deux comportements possibles face à l'augmentation de la concurrence : coopérer ou se différencier.

Enfin, peut être plus récemment, du fait de l'augmentation des enjeux stratégiques autour du phénotypage, la nature des rapports entre les acteurs impliqués dans la production et l'utilisation des données de phénotypes évolue. Ainsi, tirant parti de leur implantation géographique commune et des synergies entre leurs activités, les ES et les entreprises de contrôle de performances et de conseil en élevage (elles-mêmes souvent en forte restructuration notamment dans un contexte de libéralisation des services de contrôle de performances), se rapprochent. On n'observe généralement pas de fusions de structure, mais plutôt des accords contractuels pour le développement d'activités et de mise à disposition de moyens ou de données. En effet, le contrôle de performances est devenu une entreprise à part entière, avec la volonté de valoriser les données produites, en particulier les données de type nouveau non réglementées (données de santé ou de composition fine du lait, par exemple). De leur côté, comme nous l'avons vu précédemment, les ES ont besoin de nouveaux phénotypes à prendre en compte en sélection et des populations de référence correspondantes. L'analyse reste à approfondir quant au positionnement des entreprises de conseil en élevage.

\section{3 / Les rapports entre les acteurs de la sélection et les éleveurs}

La sélection génomique participe également à une évolution des rapports entre éleveurs et entreprises de sélection, tant au niveau de leur participation à la création de progrès génétique qu'au niveau de l'utilisation des biens et services proposés par les entreprises. Du côté de la création de progrès génétique et du rôle des éleveurs sélectionneurs dans celle-ci, la sélection génomique rend possible pour les ES la recherche de taureaux dans une plus grande diversité d'élevages et non plus uniquement chez les éleveurs les plus impliqués historiquement, fournisseurs habituels des taureaux aux schémas de sélection. Par ailleurs, la temporalité de l'évaluation des jeunes taureaux évoluant, ceux-ci obtiennent maintenant des index génomiques alors qu'ils sont encore chez les éleveurs. Cela implique certaines modifications dans les contrats de sélection quant à la propriété des taureaux et de leurs index, le temps qu'ils restent chez les éleveurs.

On observe également une évolution de l'offre génétique. De nouveaux « biens » et services sont apparus depuis 2009, qu'il s'agisse des jeunes taureaux avec index génomique ou du service de génotypage des femelles. Passer de la commercialisation de doses de taureaux avec index sur descendance à celle de doses de taureaux avec index génomique, est-ce vraiment différent? Cela a-t-il des implications autres que le simple remplacement presque invisible pour les éleveurs d'une valeur par une autre? Pour l'instant, les premiers entretiens réalisés révèlent une perte de repères dont il faudra étudier la persistance ou non. Les éléments tirés des projets présentés ici mettent en avant des usages et réactions très variés vis-à-vis de l'index génomique, allant de la méfiance à une confiance sans mesure. Ainsi, des acteurs rencontrés font part d'un manque de confiance potentiel de la part de certains éleveurs, notamment ceux pour lesquels le nombre de filles de testage était pris en compte dans leurs décisions. Il s'agit, en effet, d'un mode d'utilisation des taureaux qui nécessite des apprentissages. Une autre crainte exprimée est celle d'une utilisation des doses de taureaux avec index génomique sans prise en compte de la fiabilité de ces index (exprimée par le Coefficient de Détermination), qui est un peu moindre pour les index génomiques qu'après testage sur descendance, notamment pour les caractères de production. "Il y a un risque [...] comme on l'a vu pour les caractères de production [...] qu'on survalorise la qualité des informations utilisées. On donne plus d'importance aux informations génomiques parce que c'est à la mode [...]. Le risque, y compris en allaitant, est que les gens se concentrent trop sur cette information génomique et soient déçus à cause du manque de précision » (entretien responsable génétique ES, 2012). Des préconisations sont faites aussi bien par les scientifiques que par ceux qui réalisent chez les éleveurs les plans d'accouplement, comme par exemple celle de " ne pas mettre tous ses oufs dans le même panier » et d'encourager l'utilisation d'une large gamme de taureaux lorsque ceux-ci n'ont pas encore été évalués sur descendance. Mais le développement et l'utilisation d'un nouvel indicateur est une période d'incertitude qui entraîne des apprentissages qui peuvent être accompagnés ou non. Il sera nécessaire d'analyser plus précisément la façon dont les éleveurs s'en saisissent lorsque les index génomiques seront devenus un outil habituel.

Il est nécessaire d'être attentif à cette période d'incertitude envers ce nouvel outil, car certaines entreprises étrangères commercialisant de la semence peuvent «surfer» sur la vague d'une potentielle crise de confiance, voire l'entretenir en partie, pour se démarquer des opérateurs historiques français auprès des éleveurs. Pour cela, ils élaborent un discours à la fois technique et commercial. Ils mettent en avant la fiabilité moins bonne des index génomiques par rapport aux index sur descendance, et l'importance de n'utiliser principalement que des taureaux testés sur descendance. Ils construisent ainsi des démonstrations techniques visant à former leurs commerciaux sur ce qu'ils considèrent comme les limites de l'évaluation génomique et la différence de stratégie de leur pays d'origine, présentée comme plus prudente, par rapport à la France : "Ça n'est pas bon pour notre image de marque quand un taureau ne confirme pas. [...] 
J'insiste là-dessus au moment la vente. Nous souhaitons garder nos éleveurs et vendre plusieurs fois chez eux. Je conseille plutôt les taureaux testés. [...] On vend quand même $20 \%$ à peu près de doses de jeunes taureaux. Mais en France ils en sont à $80 \%$ (...) on ne veut pas en faire plus » (entretien commercial entreprise étrangère, mai 2013).

Alors que nous sommes en période transitoire, ces éléments permettent de pointer du doigt l'importance qu'il y a aujourd'hui de prendre en compte ce qui se joue en termes de discours et d'apprentissages relatifs aux index génomiques ou plus généralement à la sélection génomique dont les index sont un outil. Cela est d'autant plus essentiel dans un contexte où l'apparition d'indicateurs non officiels est possible et alors même que l'origine des index officiels est souvent invisible pour l'utilisateur final. Quelle connaissance les usagers finaux ont-ils du circuit de production des index entre les organismes de contrôle de performances, l'INRA et l'Institut de l'Elevage?

Comme Colleau et al (2009) l'ont indiqué au travers de simulations, la gestion des accouplements sous le « régime de la génomique » ne doit plus se faire selon la logique du « star system ». Le «star system » était la pratique par laquelle les éleveurs privilégiaient l'achat de doses provenant d'un très petit nombre de taureaux, considérés comme des « stars » nationales voire internationales pendant plusieurs années. Ce système ${ }^{9}$ est apparu avec le développement de l'insémination artificielle, qui a permis de diffuser largement certains taureaux. Il a eu des effets négatifs importants sur la variabilité génétique, sur l'apparition d'anomalies génétiques, sur la dégradation de certains caractères non sélectionnés. Il faut maintenant profiter pour les bovins laitiers du fait que les jeunes taureaux avec index génomiques mis en marché ont une précision moindre et qu'un plus grand nombre de jeunes taureaux peuvent être commercialisés, pour favoriser l'emploi au niveau de chaque éleveur de plus de taureaux et ainsi protéger le bien commun. Les premiers entretiens révèlent que ce changement de pratique n'est pas toujours facile à mettre en œuvre. Certains parlent de choc culturel. Les informations sur les taureaux et les taureaux eux-mêmes connaissent un turn-over beaucoup plus rapide qu'auparavant. Certains techniciens rencontrés nous ont ainsi fait part de leurs difficultés à se " tenir à jour », notamment ceux qui ne sont pas spécialisés uniquement en sélection comme les techniciens des organismes de contrôle laitier, mais ces questionnements sont aussi apparus auprès des inséminateurs. Ils expriment ces difficultés également ressenties par les éleveurs : "Pour moi, ça n'est pas compliqué : je suis complètement dépassée. Je trouve qu'avoir une série de nouveaux taureaux tous les trois mois est très difficile à gérer pour nous au quotidien. Avant la génomique, nous faisions quelques plannings d'accouplements mais on en fait pratiquement plus aujourd'hui. [...] On est perdus, on a décidé de laisser tomber " (entretien collectif techniciens ECEL, avril 2013)

Il serait nécessaire d'analyser les évolutions, à plus long terme, de cette « anonymisation » des taureaux ${ }^{10}$ et de cet accroissement du turn-over des données, à la fois sur les pratiques des éleveurs pour la sélection de leur troupeau, et sur la redéfinition des compétences et des rôles des différents opérateurs impliqués dans le conseil aux élevages. Quels seront les sélectionneurs et les utilisateurs de demain? Quelles seront leurs pratiques vis-à-vis de ces nouveaux services ? Ces questionnements devront être traités en prenant en compte l'évolution plus générale des systèmes d'élevage, des filières, dans un contexte de suppression des quotas et d'agrandissement des cheptels. En effet, dans d'autres domaines tels que les télécommunications par exemple, la libéralisation des services couplée au développement de nouvelles technologies a conduit à l'évolution des identités : «l'usager » est devenu « consommateur » (Fijalkow 2006). Dans le cas de la sélection des ruminants, les éleveurs sont pour l'instant à la fois usagers et producteurs du progrès génétique. Il est important d'analyser ces changements dans les identités professionnelles des éleveurs (éleveur-sélectionneur, éleveurutilisateur...). Cela implique, par exemple, de repenser l'accompagnement de la gestion de la reproduction des troupeaux, d'imaginer la construction de logiciels d'accouplements adéquats, d'analyser les usages des nouveaux services tels que l'évaluation génomique des femelles et d'adapter les contrats de sélection à ces changements de pratiques des éleveurs vis-à-vis des produits et services génétiques.

\section{4 / Des évolutions dans la gou- vernance des races?}

Les Organismes de Sélection (OS) sont définis par la Loi comme étant la structure en charge de la gouvernance des races. Les différents acteurs impliqués dans la gestion et la sélection s'y retrouvent pour définir les objectifs de sélection communs et partagés, et pour assurer la promotion de la race. Couplée à la mutualisation des activités de recherche-développement et de l'évaluation, les offres génétiques par les Entreprises de Sélection étaient donc relativement peu distinctes. Comme nous l'avons vu précédemment, la sélection génomique rend possible le développement de stratégies différenciées de la part des entreprises de sélection en leur permettant de gérer beaucoup plus finement le choix de leurs taureaux et de leurs mères à taureaux, mais également au travers de la constitution d'offres spécifiques sous forme de « profils » de taureaux. Des ES cherchent également à développer des objectifs de sélection qui leur sont propres, au travers d'investissements privés de recherche dans du phénotypage et la création de populations de références privées (elles produisent ainsi leur propre index de synthèse, ce qui va au-delà de la simple segmentation de l'offre en « profils » de taureaux). Ces dynamiques amènent certains acteurs représentant des OS (dont font partie les ES) à s'interroger sur l'évolution du rôle de ces organismes et de ce qui était reconnu comme le «parlement de la race ». Qu'advient-il de la construction de stratégie de sélection commune dans le contexte d'un développement de stratégies individuelles ? La place de ces structures communes que sont les OS dans le développement de la sélection génomique est complexe, car tandis que ces organismes ont peu de moyens financiers à proprement parler, leur rôle d'orientation de la race et de représentant des propriétaires collectifs de la race et de ses usagers (éleveurs, sélectionneurs, filières d'aval, territoires) est important. En effet, leur rôle est indispensable pour assurer l'adhésion et la coopération avec l'ensemble des acteurs d'une race, des éleveurs et associations d'éleveurs jusqu'aux usagers de l'aval, autour de la race comme bien commun et comme institution. Ainsi, le responsable de l'un des OS rencontrés a évoqué les difficultés de ce parlement racial à pouvoir « arbitrer pour l'intérêt collectif » face à des stratégies différenciées entre les ES et une augmentation de la concurrence entre elles. L'analyse de l'évolution de la gouvernance des races, processus sur le temps long, reste à approfondir dans la suite des projets de recherche présentés ici.

Ces analyses donnent quelques exemples des reconfigurations des rapports entre acteurs dans le cas d'une rupture technologique profonde, associée à la libéralisation d'un système. Ainsi, on ne peut pas simplement penser qu'il y a « plus » ou « moins » de coopération à

\footnotetext{
${ }^{9}$ Qui n'existe pas en ovins laitiers, du fait de l'utilisation de semence fraiche.

10 On se rapprocherait alors du système utilisé pour les ovins laitiers.
} 
l'ère de la sélection génomique, mais il est en revanche assez clair qu'il y a une redéfinition du paysage organisationnel de la sélection, des rôles et des compétences des acteurs, et de leurs identités professionnelles. Jusqu'en 2006, les relations de coopération entre les acteurs étaient relativement clairement établies, avec les nombreuses règles définies dans la Loi sur l'élevage de 1966. Depuis 2006, puis 2009 , ces règles ont changé, et le contexte technologique aussi : les champs d'innovation sont nouveaux, les acteurs se retrouvent dans une période de forte incertitude aussi bien sur les connaissances (qui sont à construire) que sur les relations (qui sont à (re)construire aussi ou à faire évoluer). D'un côté, il y a nécessité de plus de coopération pour compenser des investissements lourds de recherche-développement. De l'autre, il existe moins de règles collectives, plus de compétition, mais davantage « d'opportunités » pour l'individualisme dans un contexte plus compétitif. Les différents cas observés en bovins laitiers et allaitants révèlent la diversité des stratégies des acteurs face à ce contexte. L'évolution du contexte économique de l'élevage influence aussi forcément ces stratégies, notamment concernant les attentes des éleveurs dans un contexte de fluctuation des prix.

\section{3 / Accompagner les change- ments organisationnels : le cas des ovins laitiers}

Les acteurs de la sélection des ovins laitiers dans les trois bassins de production (Roquefort, Pyrénées-Atlantiques et Corse) ont pu observer, de l'extérieur, les bouleversements récents apparus dans le secteur de la génétique bovine laitière. Dès 2009, les bassins de Roquefort et des Pyrénées-Atlantiques se sont parallèlement engagés dans des programmes de recherche pour tester la faisabilité de la sélection génomique des ovins laitiers (Roquefort'in, Genomia) (Duchemin et al 2012, Baloche et al 2014). Ils ont rapidement pris conscience que tout en développant les aspects «techniques » de la génomique, il était indispensable de réfléchir aux évolutions organisationnelles nécessaires au développement de cette technologie ou induites par celleci, afin de les accompagner. Les acteurs des trois bassins se sont interrogés sur la façon dont ils pouvaient se préparer à ces changements afin d'assurer le maintien des capacités collectives de recherche, de développement et de sélection qui ont assuré l'efficacité des schémas de sélection jusqu'à présent, fondés sur une gestion pyramidale de la population en sélection avec une dichotomie claire entre éleveurs sélectionneurs et utilisateurs. Une action innovante de FGE a ainsi été mise en place, permettant d'organiser une réflexion en amont sur les conséquences potentielles de la sélection génomique sur les collectifs de sélection, et les manières de les anticiper pour assurer le maintien de ces collectifs. Les trois bassins ovins laitiers ont été impliqués, sachant que ce sont principalement le Rayon de Roquefort et les PyrénéesAtlantiques qui sont concernés à court terme.

Le projet COSEGOV porté par le Comité National Brebis Laitières (action innovante bénéficiant d'un financement de FGE) ${ }^{11}$ a reposé sur une méthode de recherche innovante. Dans un premier temps, nous avons organisé des ateliers de réflexion collectifs, réunissant dans chaque bassin les responsables techniques, administratifs et professionnels des schémas de sélection, les ingénieurs de l'Institut de l'Elevage et les chercheurs de l'INRA. Ces ateliers étaient organisés en deux temps : un premier temps de partage de connaissances autour de la génomique et de la méthode d'évaluation génomique en elle-même, mais aussi autour du fonctionnement organisationnel actuel des schémas et de leur évolution en version génomique ; un deuxième temps de réflexions dirigées où les participants ont pu échanger autour de différentes thématiques (la place des sélectionneurs et des utilisateurs, la diffusion des index génomiques, la gestion des populations de référence, les contrats de sélection...) et identifier les risques et opportunités de la génomique. Au total, 14 heures d'ateliers ont été enregistrées et analysées. Dans une deuxième phase, des entretiens individuels ont été réalisés auprès d'une diversité d'acteurs dans chaque bassin (éleveurs sélectionneurs en contrôle laitier officiel, éleveurs utilisateurs hors contrôle laitier officiel, responsables d'OS, responsables et techniciens d'ES). L'objectif était d'approfondir les thèmes ayant émergé des ateliers collectifs et d'obtenir des informations et des points de vue qui n'auraient pas été abordés durant la phase collective. Ainsi, 15 entretiens ont été réalisés dans la zone de Roquefort (race Lacaune), 9 en Pyrénées-Atlantiques (races ovines laitières des Pyrénées) et 1 en Corse (race Corse).

Trois axes de réflexion sur les changements à anticiper sont fortement ressortis des entretiens et des ateliers : le maintien de l'action collective, l'amont de la production d'index génomiques et de béliers avec index génomiques, et l'aval de cette production avec la diffusion de ces index et de ces béliers (Rousseau 2012).

\section{1 / Accompagner et maintenir l'action collective}

L'enjeu de maintenir l'action collective de sélection (cf. encadré) et la coopération entre l'ensemble des acteurs (qu'il s'agisse des éleveurs sélectionneurs ou utilisateurs, des entreprises de sélection, des OS ou de la recherche) a été clairement réaffirmé dans les discours : «on veut resserrer le collectif $\gg$ (responsable $\mathrm{ES}$ ), face au risque de développement de circuits de diffusion parallèles et privés qui menaceraient l'efficacité des schémas. En effet, plus encore que chez les bovins, l'engagement financier important que représente la génomique ne peut fonctionner à long terme que si les collectifs se maintiennent et que les comportements individualistes sont limités.

Pour maintenir l'action collective, les acteurs rencontrés considèrent qu'il faut à la fois maintenir l'engagement des éleveurs sélectionneurs, dont le travail est nécessaire pour la production et la collecte des phénotypes, et l'entretien de la population de référence (qui remplacera le testage sur descendance du schéma actuel), et s'assurer de la durabilité de la collaboration avec la recherche-développement (collaboration qui se matérialise au sein du Comité National Brebis Laitières, association loi 1901 fédérant les organismes du dispositif génétique ovin lait et qui représente au sein de FGE et de sa commission ovine la filière ovin lait).

Concernant l'engagement des éleveurs sélectionneurs, il est nécessaire de maintenir les incitations individuelles pour assurer la participation au collectif. Dans le cas des éleveurs en CLO, il s'agit de préserver la supériorité génétique moyenne de leurs troupeaux par rapport aux troupeaux des éleveurs utilisateurs (en contrôle laitier simplifié ou hors contrôle laitier) : en pratique les IA en semence fraîche (avec des lots moyens de 150 à 200 brebis inséminées par élevage et par jour) signifient qu'aucun éleveur ne choisit ses béliers d'IA. Le pack de béliers d'IA (nombre de béliers et de doses par bélier) par élevage est donc planifié de telle façon que la valeur génétique soit comparable entre sélectionneurs et supérieure à la valeur génétique du pack de béliers d'IA pour les élevages utilisateurs, la différence entre les deux types d'élevage correspondant à un écart de 3-4 ans. Autrement dit, le schéma de

\footnotetext{
11 COSEGOV, 2013, « Refondation des Contrats de Sélection et d’Utilisation en Ovin en lien avec l'émergence de la Génomique ». Compte-rendu final, Convention $\mathrm{n}^{\circ} 611$ fge $043,14 \mathrm{p}$.
} 
Encadré. Organisation des schémas de sélection en ovins laitiers.

Contrairement aux bovins laitiers, la spécificité des schémas de sélection en ovins laitiers est d'être fondée sur une gestion pyramidale de la population, avec un noyau de sélection d'environ $20 \%$ de la population à améliorer (10 à $30 \%$ en fait selon sa taille), composé d'éleveurs sélectionneurs chez qui sont concentrés tous les outils nécessaires à la sélection à la fois des béliers pour l'Insémination Artificielle (IA) et pour la Monte Naturelle (MN) requis pour tous les éleveurs, sélectionneurs et utilisateurs (Barillet 1997) : les éleveurs sélectionneurs en Contrôle Laitier Officiel (CLO) s'engagent à réaliser la mesure des phénotypes de tous les caractères sélectionnés, la gestion des paternités en IA et MN, le testage des béliers d'IA à hauteur d'environ $50 \%$ des IA et les accouplements raisonnés pour la procréation des béliers d'IA et de MN nécessaires aux besoins de toute la population. Une fraction importante de ces jeunes mâles nés d'accouplements raisonnés entre en centres d'élevage de jeunes mâles, pour approvisionner les centres d'IA ou être diffusés comme béliers de MN chez les éleveurs utilisateurs. L'engagement des éleveurs sélectionneurs en CLO est coûteux en temps et représente un investissement financier important, compensé par le fait qu'ils bénéficient toujours en priorité du progrès génétique produit par le schéma, par exemple en privilégiant les IA avec les béliers élites (Pères à Béliers-PAB) chez les sélectionneurs.

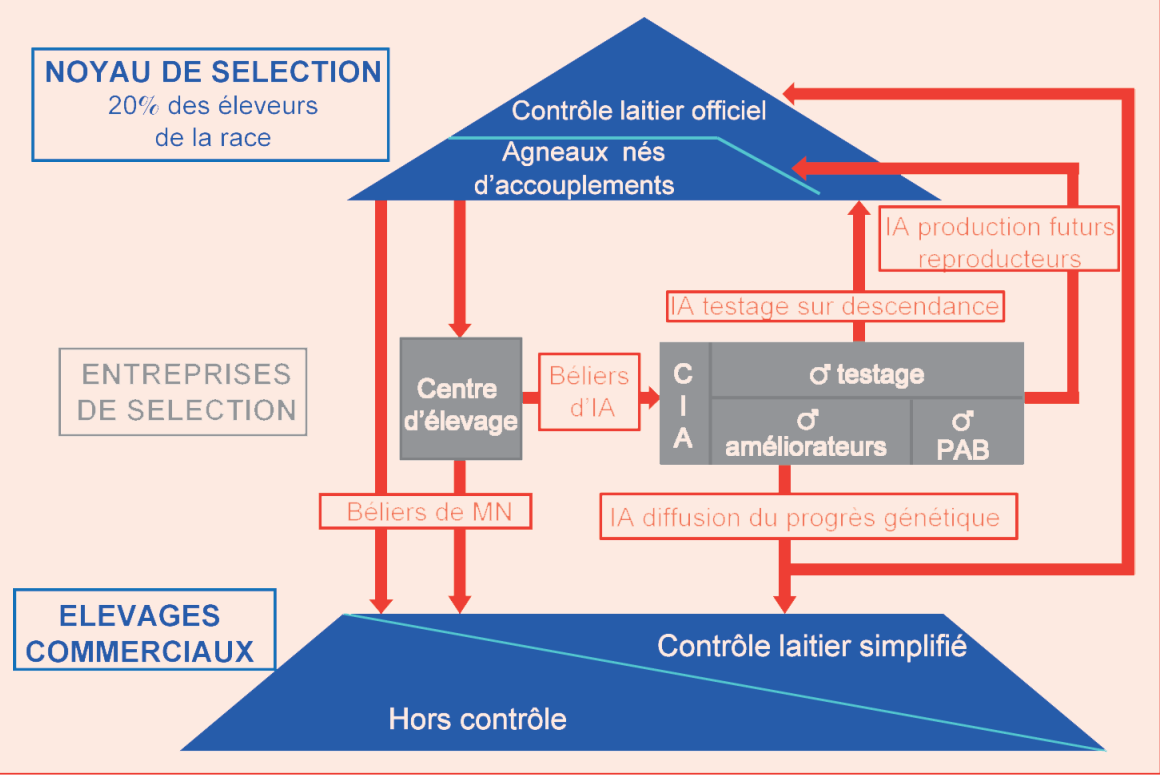

sélection pyramidal actuel, bien loin du «star système » décrit auparavant en bovins laitiers, mime déjà parfaitement la stratégie génomique de packs de mâles d'IA assurant le maintien de la diversité génétique et gérant au mieux la prise de risque sur la valeur finale des animaux (« pas tous les œufs dans le même panier »). C'est donc de façon consciente que les éleveurs sélectionneurs veulent préserver cette réalité, la sélection génomique ne remettant pas en cause cette organisation pyramidale, d'autant que l'arrivée de la génomique pourrait impliquer le développement de nouvelles tâches pour ces éleveurs sélectionneurs : garder les agneaux plus longtemps sur leur exploitation dans l'attente des résultats des génotypages, ou réaliser de nouvelles mesures de phénotypes pour de nouveaux caractères à sélectionner. Les acteurs rencontrés ont ainsi mis en avant la nécessité de bien mesurer ces nouveaux investissements individuels à réaliser de la part des éleveurs sélectionneurs, et d'assurer une contrepartie suffisante pour maintenir leur engagement dans les schémas de sélection.
Concernant la coopération entre les acteurs de terrain et les acteurs de la recherche et du développement, l'analyse des ateliers et des entretiens a mis en avant la nécessaire adéquation entre les moyens d'ingénierie et les outils mis à disposition par l'INRA et l'Institut de l'Elevage dans le cadre de l'UMT GGPR, avec un sentiment, de la part des acteurs de la recherche des réalités de celui-ci. L'une des craintes identifiées a été le risque d'accroissement de l'écart d'investissement scientifique entre bovins et ovins : la recherche publique va-t-elle continuer à investir dans les filières ovines ? Va-t-elle continuer à investir dans les filières qui n'auront pas développé la sélection génomique?

\section{2 / Organiser la production d'index génomiques}

Le deuxième axe thématique a été l'organisation amont de la production d'index génomiques et la gestion de béliers avec ou sans index génomiques, de terrain, d'un éloignement progressif sachant que le noyau de sélection produit chaque année à la fois des béliers d'IA et de MN (figure 2).

L'apparition de nouvelles données (les index génomiques) pose la question des droits de propriété associés. La gestion des agneaux en attente de génotypage est un aspect important de la réorganisation des centres de sélection. Les enjeux organisationnels sont différents selon les modalités de fonctionnement actuelles de chaque entreprise de sélection. Ainsi, en races Lacaune et Pyrénéennes, les trois entreprises de sélection (Confédération de Roquefort et Ovitest pour la race Lacaune, $\mathrm{CDEO}^{12}$ pour les races Pyrénéennes) ont des modes de gestion des agneaux différents. Pour l'une des ES Lacaune, tous les agneaux mâles nés d'accouplements raisonnés chez les sélectionneurs appartiennent à l'ES et intègrent le centre d'élevage (ceux destinés au centre d'IA et également les futurs béliers de monte naturelle) qui a une capacité importante : les agneaux qui seront génotypés attendront donc la publication de leurs index génomiques dans le centre d'élevage. En revanche, pour l'autre ES Lacaune, n'entre en centre d'élevage que le nombre d'agneaux nécessaire au besoin pour le testage. Une fois la sélection génomique mise en œuvre, cette ES devra s'organiser pour faire entrer plus d'agneaux en centre d'élevage qu'actuellement de façon à pouvoir exercer la pression de sélection génomique requise, avec les limites d'ordre structurel (capacité limitée des bergeries). Enfin, dans l'ES des Pyrénées, pour faire entrer l'ensemble des agneaux à génotyper en centre d'élevage, il faudrait investir dans de nouvelles bergeries : la solution envisagée est de laisser les agneaux chez les éleveurs dans l'attente des index génomiques, mais cela implique une gestion différente de ces agneaux par les éleveurs, et des contrats différents avec l'ES pour compenser la charge que représente l'entretien de l'ensemble des agneaux candidats nécessaires durant cette période.

La sélection génomique implique pour ces dispositifs collectifs de financer le coût du génotypage d'un nombre suffisant d'agneaux permettant d'exercer une pression de sélection génomique en rapport avec le gain génétique recherché. Or, contrairement au cas des bovins laitiers, les économies financières possibles en retour sont moindres. Du fait de la distribution en semence fraiche, les entreprises ont l'obligation de disposer d'un cheptel de béliers en centre d'insémination animale suffisant pour la diffusion, et ce d'autant plus que la période de reproduction est très saisonnée. $\mathrm{La}$ réduction du nombre de mâles dans les 
Figure 2. Différentes catégories possibles de béliers avec index génomiques (issu de Rousseau 2012).

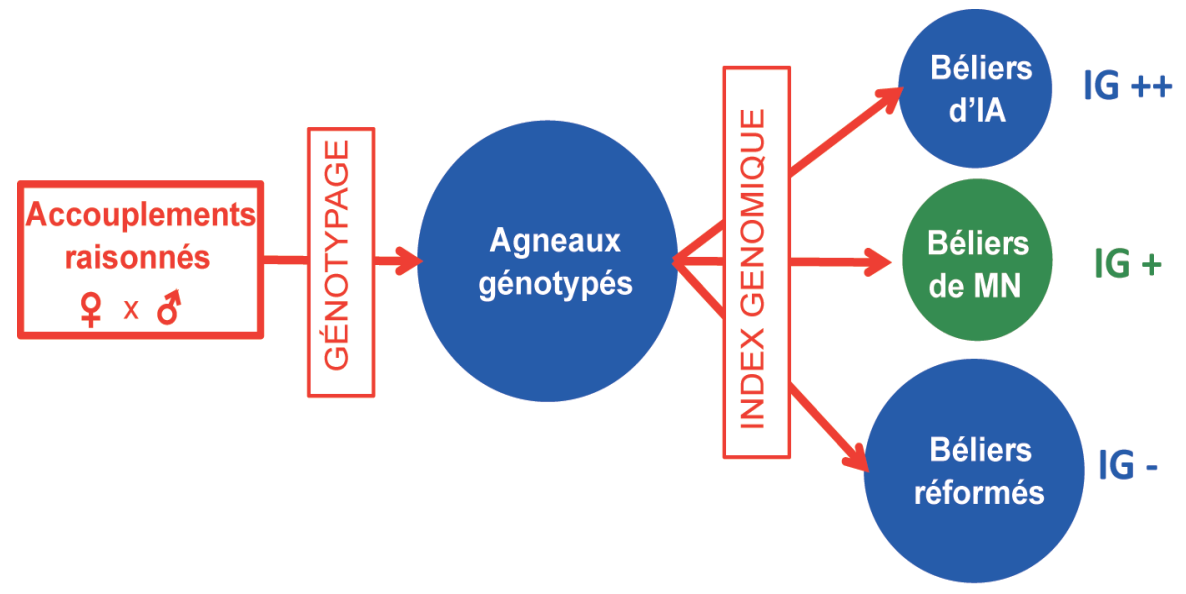

centres d'IA sera donc moins importante, mais constitue tout de même un élément clé de l'équilibre technico-économique de la sélection génomique en ovin laitier. Le contrôle de performances ne pourra également pas être réduit, puisque c'est déjà le cas avec l'organisation pyramidale. Les cycles biologiques des ovins étant plus courts, la réduction de l'intervalle de génération avec la sélection génomique est négligeable à l'inverse des bovins, et la précision des index génomiques chez les ovins laitiers sera également moindre que chez les bovins laitiers. L'ensemble de ces aspects entrâ1nera une augmentation du gain génétique moins importante qu'en bovins laitiers. De plus, la puce ovine est plus chère que la puce bovine, alors que la valeur financière d'un bélier est moindre que celle d'un taureau. Le prix du génotypage d'un bélier est donc plus élevé, et les béliers sont plus nombreux dans les entreprises de sélection. Tous ces paramètres font que la supériorité technique et économique de la sélection génomique sur la sélection classique, très forte en bovin, est comparativement plus faible en ovins. La mise en place de la sélection génomique pour les ovins laitiers implique donc de calibrer un schéma de sélection génomique au plus juste en optimisant un compromis entre gain génétique et réduction du nombre de béliers en centre d'IA (par suppression de la période d'attente avant connaissance de la valeur génétique sur descendance et optimisation de la gestion des béliers) pour compenser les coûts liés au génotypage. Bien sûr, une réduction significative (espérée) des coûts de génotypage permettrait d'augmenter la pression de sélection et donc le gain génétique de +15 à $30 \%$ par rapport au schéma actuel, tout en conservant un bon compromis technicoéconomique. La question de l'optimisation de la capacité des béliers d'IA à produire de la semence de qualité est également au cœur de l'équilibre économique du schéma génomique.

\section{3 / Gérer la diffusion des index génomiques}

Une fois les index génomiques obtenus, comment gérer ces résultats et leur diffusion? C'est le troisième axe thématique abordé dans le projet COSEGOV, autour duquel les débats lors des ateliers et les entretiens ont soulevé d'importantes questions. Il est bon de rappeler ici que la monte naturelle en ovins laitiers est importante, même lorsque l'IA est développée, car il n'y a qu'une IA (IA sur lots de brebis synchronisées), les retours étant assurés par des béliers de monte naturelle : il y a donc toujours des béliers de monte naturelle (de l'ordre de 4 à 8 par troupeau) dans les élevages. Le recours à l'IA ne concernant que $44 \%$ des brebis laitières en France (à comparer à $94 \%$ des vaches laitières et $4 \%$ des ovins viande) $)^{13}$, il y a donc un marché de reproducteurs de monte naturelle en ovins laitiers. Une partie des agneaux avec un bon index sur ascendance classique (nés d'accouplements raisonnés), qui aurait donc été valorisée dans le schéma classique, aura après génotypage des index génomiques moyens à faibles. Pour préserver la confiance des éleveurs envers les schémas de sélection, ces agneaux ne pourront être vendus et devront être éliminés, ou alors vendus à un prix inférieur, ce qui là encore ajoutera un coût certain au schéma. Dans le cas où les agneaux restent la propriété des sélectionneurs, comment gérer la diffusion de l'information « index génomique »? Celle-ci doit-elle être diffusée ? Les sélectionneurs risquent-ils de décider d'utiliser leurs « bons béliers » au détriment de l'insémination artificielle? Les acteurs rencontrés dans le Rayon de Roquefort ont indiqué qu'ils étaient confiants sur le maintien de l'utilisation de l'insémination artificielle au niveau du bassin. En revanche, il est fréquemment ressorti des ateliers et des entretiens le risque que ces béliers avec index génomique puissent fortement intéresser les acheteurs hors bassin ou étrangers. Deux stratégies ont alors été envisagées et discutées : soit ne pas communiquer l'index génomique, aussi bien au niveau national qu'international, mais cela bloque toute possibilité de retour sur investissement de la génomique (impossibilité de vendre plus cher des béliers de $\mathrm{MN}$ avec de bons index génomiques); soit communiquer l'index génomique pour vendre les béliers à un prix plus élevé, et assurer ainsi une plus-value pour le dispositif collectif. Mais cette deuxième stratégie comporte des risques concernant la gestion des exportations à l'étranger. Si l'index génomique est communiqué au niveau national, il est impossible de ne pas le fournir au niveau international, des éleveurs ou d'autres intervenants pouvant par exemple développer un marché parallèle de vente de béliers avec index génomique à l'étranger. De plus, la maîtrise du marché de l'export et l'avance du schéma de sélection Lacaune seraient peut-être plus difficiles à conserver si les importateurs pouvaient avoir une meilleure connaissance des valeurs génétiques des béliers de MN achetés, à condition d'en faire un bon usage. D'une part il se pourrait qu'ils réclament de meilleurs béliers, d'autre part ils pourraient peutêtre développer plus facilement leur propre schéma grâce à cette meilleure connaissance de la valeur des béliers.

Dans les Pyrénées-Atlantiques, les risques de développement de la monte naturelle au détriment de l'IA avec une meilleure connaissance de la valeur génétique des béliers de $\mathrm{MN}$ sont plus importants. En effet, contrairement au Rayon de Roquefort où les éleveurs perçoivent l'IA comme un outil de gestion de l'élevage aux multiples intérêts (facilité de travail, synchronisation des lots...) et où l'IA est un outil de maîtrise de l'étalement de la production imposé par la filière, dans les PyrénéesAtlantiques l'IA est encore perçue surtout comme un outil génétique. La concurrence entre IA et béliers de MN avec index génomique pourrait donc être plus forte.

Il est donc important de réfléchir à la forme et aux conditions sous lesquelles les index génomiques, particulièrement ceux des béliers de MN, seront diffusés. L'index génomique en tant que tel serat-il diffusé quelle que soit sa valeur ? S'agira-t-il d'un système de classement avec des fourchettes de valeur? Ou bien uniquement de la garantie que lorsqu'un

13 Données FGE 2013. 
bélier est proposé (au travers de ses doses de semence ou bien pour la vente), son index génomique soit positif? Il est également nécessaire de définir dans les contrats de sélection la propriété et l'utilisation des béliers avec index génomique. Jusqu'à présent, l'absence d'information précise sur les différences de valeur génétique entre béliers de $\mathrm{MN}$ (tous annoncés comme bons car nés d'accouplements raisonnés) réduisait, notamment dans le bassin de Roquefort où les index génétiques sont considérés comme une référence partagée (Labatut et al 2012), l'intérêt des ventes de gré à gré comparativement à l'IA. Avec l'indexation génomique, ces béliers auront des informations plus précises (grâce à l'investissement collectif dans l'évaluation génomique), qui pourront potentiellement faciliter leur utilisation sur le marché de la monte naturelle. Cependant, il est pour l'instant difficile d'anticiper la façon dont ces index génomiques seront perçus par les éleveurs et les usages qui émergeront. Il s'agira alors d'assurer un suivi de ce nouvel outil d'évaluation parallèlement à l'accompagnement des éleveurs, pour adapter au fur et à mesure les règles de diffusion et d'usage. L'un des enjeux importants restera malgré tout d'homogénéiser ces règles entre bassins de production, car si dans l'un des bassins les informations génomiques sont plus accessibles que dans un autre, cela pourra rapidement créer des précédents et entraîner des difficultés. C'est également au niveau des échanges entre pays que le statut et les règles de diffusion de ces informations devront être établis. Ces règles sont en cours de modification au niveau européen et les conclusions du projet COSEGOV doivent être l'opportunité pour les ovins d'être force de propositions au niveau des instances concernées par l'évolution de cette réglementation. Cette discussion devra se faire entre les trois bassins ovins laitiers, mais également en concertation avec les ovins allaitants.

La diffusion des index génomiques concerne aussi la question de l'éventuel développement d'un service de génotypage, tel que celui-ci est apparu dans la filière de la sélection bovine laitière. Mais il s'agirait dans le cas des ovins non pas d'un service de génotypage pour les femelles puisque le coût du génotypage par rapport à la valeur d'une brebis serait pour l'instant beaucoup trop élevé (même si les enquêtes ont montré qu'avec un coût inférieur à 10 euros, la possibilité du génotypage des femelles serait à reconsidérer), mais d'un service de géno- typage pour les mâles de MN. Mais encore une fois, la plupart des acteurs enquêtés voient dans l'ouverture potentielle de ce service un risque fort de déstabilisation des collectifs, en favorisant les comportements individualistes au travers de la création d'un marché parallèle de reproducteurs évalués génétiquement.

\section{Conclusion}

Les deux projets ont permis d'identifier et d'explorer différents changements en cours, aussi bien au niveau de l'élaboration de l'évaluation génétique et de la production de données, qu'à celui de la diffusion de nouveaux biens et services génétiques pour les bovins laitiers. Les rôles et les compétences des différents acteurs de la sélection sont redéfinis et ceux-ci développent des stratégies différenciées dans un contexte de plus en plus concurrentiel. L'accélération de la production d'informations sur les animaux et le turn-over beaucoup plus rapide des taureaux reproducteurs bouleverse les pratiques de gestion du renouvellement des troupeaux. Pour les ovins laitiers, ces résultats ont permis d'anticiper certains changements en amont de la mise en œuvre de la sélection génomique, et d'identifier les outils et dispositifs à mettre en place pour s'assurer du maintien des capacités collectives de sélection.

$\mathrm{Au}$ fur et à mesure du développement et de la diffusion de la sélection génomique, des aspects particuliers sont devenus des enjeux de plus en plus forts pour les acteurs de la sélection. C'est le cas de la production des données de phénotypage (Lagarrigue et Tixier-Boichard 2011) et de la construction des populations de référence. Ces données deviennent aujourd'hui un nouvel enjeu de pouvoir entre les acteurs, alors qu'elles avaient toujours été mutualisées avant la sélection génomique. Certains acteurs deviennent stratégiques pour certains types de données. De nouveaux acteurs ne participant pas auparavant aux activités génétiques peuvent y trouver un intérêt. Cet enjeu et cet engouement autour des données n'avaient pas vraiment été anticipés. Il est donc aujourd'hui crucial d'analyser ces dynamiques de production et de flux de données, et de s'intéresser à la question du partage et des droits de propriétés autour de cellesci, notamment dans un contexte de changement politique important à venir (projet de règlement européen sur l'organisation de la sélection génétique en 2017).
Parallèlement, il est nécessaire de continuer à identifier l'évolution des pratiques et des usages inattendus de l'évaluation génomique et de ses produits (index génomiques) chez les acteurs de la sélection au quotidien (éleveurs, inséminateurs, contrôleurs laitiers, techniciens et responsables de stations d'évaluation, acteurs de la recherche-développement...) et leurs conséquences sur les marchés des biens et des services, des animaux et de leurs semences. Comme nous l'avons vu, les changements identifiés dans le cadre des projets STRING et de COSEGOV sont vraisemblablement transitoires. Quelles formes vont prendre les pratiques stabilisées ? L'identité de l'objet « index génétique/génomique » est par exemple questionnée par le fait qu'il devienne un objet marchand (service de génotypage) et par le fait que ses modes et circuits de production évoluent (diversification des sources de données). L'éventualité de l'ouverture du service de génotypage aux taureaux hors entreprises de sélection après 2015 sera un facteur très important à prendre en compte dans l'analyse de ces changements et de la déstabilisation possible des dispositifs coopératifs de sélection et des stratégies que les acteurs vont développer face à cette ouverture. Ces deux axes de travail (gestion des données et changements de pratiques autour des outils de la sélection en ovins et bovins) font l'objet du projet Coopigen, financé par le Méta-programme SELGEN de l'INRA (2013-2015).

La sélection génomique continue donc à offrir de nombreuses perspectives aux acteurs de l'élevage. Les éléments pointés ici amènent à insister sur :

- L'opportunité d'élargir la gamme des partenariats possibles : la génomique ouvre le champ des possibles pour des acteurs non historiques dans le domaine. Il y a donc un enjeu pour les acteurs historiques à imaginer des partenariats innovants, au-delà des partenariats horizontaux, notamment pour le développement de la sélection sur de nouveaux caractères.

- La nécessité de suivre de près les évolutions scientifiques et technologiques, qui continueront à avoir des implications organisationnelles et stratégiques fortes pour les acteurs de la sélection (par exemple dans le cas des recherches menées sur l'identification de mutations causales ou délétères). 


\section{Références}

Astruc J.M., Baloche G., Larroque H., Beltran de Heredia I., Labatut J., Lagriffoul G., Moreno C., Robert-Granié C., Boscher M.Y., Chantry-Darmon C., Aguerre X., Boulenc P., Fregeat G., Giral-Viala B., Guibert P., Panis P., Soulas C., Barillet F., 2012. La sélection génomique des ovins laitiers en France : stratégies, premiers résultats des évaluations génomiques et perspectives. Renc. Rech. Rum., 19, 81-84.

Baloche G., Legarra A., Sallé G., Larroque H., Astruc J.M., Robert-Granié C., Barillet F., 2014. Assessment of accuracy of genomic prediction for French Lacaune dairy sheep. J. Dairy Sci., 97, 1107-1116.

Barillet F., 1997. Genetics of Milk Production. In: The Genetics of Sheep. Piper L., Ruvinsky A. (Eds). CAB International, 539-564.

Bidanel J.P., Boichard D., Chevalet C., 2008. De la génétique à la génomique. In : Numéro spécial, 20 ans de recherches en productions animales à l'INRA. Charley B., Herpin P., Perez J.M. (Eds). INRA Prod. Anim., 21, 15-32.

Boichard D., Guillaume F., Baur A., Croiseau P., Rossignol M.N., Boscher M.Y., Druet T., Genestout L., Colleau J.J., Journaux L., Ducrocq V., Fritz S., 2012. Genomic selection in French dairy cattle. Anim. Prod. Sci., 52, $115-120$

Bonneuil C., Thomas F., 2009. Gènes, pouvoirs et profits. Recherche publique et transformations des régimes de production des savoirs et des innovations en génétique végétale de Mendel aux OGM. Editions Quae, Paris, France, 624p.

Colleau J.J., Fritz S., Guillaume F., Baur A., Dupassieux D., Boscher M.Y., Journaux L., Eggen A., Boichard D., 2009. Simulation des potentialités de la sélection génomique chez les bovins laitiers. Renc. Rech. Rum., 16, 419.

Duchemin S.I., Colombani C., Legarra A., Baloche G., Larroque H., Astruc J.M., Barillet F., Robert-Granié C., Manfredi E., 2012. Genomic selection in French Lacaune dairy sheep breed. J. Dairy Sci., 95, 2723-2733.
Fijalkow Y., 2006. Usagers ou consommateurs? France Télécom ou la dérégulation du service public. Presses universitaires du Mirail, Toulouse, France, 314p.

Guillaume F., Boichard D., Ducrocq V., Fritz S., 2011. Utilisation de la sélection génomique chez les bovins laitiers. In : Numéro spécial, Amélioration génétique. Mulsant P., Bodin L., Coudurier B., Deretz S., Le Roy P., Quillet E., Perez J.M. (Eds). INRA Prod. Anim., 24, 363368.

Institut de l'Elevage, INRA, 2011. La révolution génomique animale. France Agricole, Paris, France, $161 \mathrm{p}$.

Labatut J., 2009. Gérer des biens communs : Processus de conception et régimes de coopération dans la gestion des ressources génétiques animales. Mines Paris Tech., 1-382.

Labatut J., 2013. Emerging markets, emerging strategies under the genomic era. EAAP, $64^{\text {th }}$ Ann. Meet. Nantes, France, 95.

Labatut J., Aggeri F., Bibé B., Girard N., 2011 Construire l'animal sélectionnable. Revue d'Anthropologie des Connaissances, 5.

Labatut J., Bibé B., Aggeri F., Girard N., 2012. Coopérer pour gérer des races locales : conception, rôles et usages des instruments scientifiques de sélection. Nat. Sci. Soc., 20, 143-156

Labatut J., Allaire G., Aggeri F., 2013a. Etudier les biens communs par les changements institutionnels : régimes de propriété autour des races animales face à l'innovation génomique. Revue de la régulation, 14

Labatut J., Dubois P., Griffon L., 2013b. Enjeux organisationnels au sein des dispositifs de sélection face au développement de la génomique : le cas de trois races bovines (Tarentaise, Brune, Aubrac). Renc. Rech. Rum., 20, 133-136.

Lagarrigue S., Tixier-Boichard M., 2011. Nouvelles approches de phénotypage pour la sélection animale. In : Numéro spécial, Amélio-

ration génétique. Mulsant P., Bodin L., Coudurier B., Deretz S., Le Roy P., Quillet E., Perez J.M. (Eds). INRA Prod. Anim., 24, 377-386.

Larivière J.M., Leroy P., 2008. Conservation et valorisation de la diversité des ressources génétiques du poulet en Europe : initiatives et perspectives. Ann. Méd. Vét., 152, 203-220.

Ostrom E., 1990. Governing the commons. The evolution of institutions for collective action. Cambridge University Press, Cambridge, 1, 280p.

Peerbaye A., 2004. La construction de l'espace génomique en France: la place des dispositifs instrumentaux. Sociologie. Ecole normale supérieure de Cachan.

Robert-Granié C., Legarra A., Ducrocq V., 2011. Principes de base de la sélection génomique. In : Numéro spécial, Amélioration génétique. Mulsant P., Bodin L., Coudurier B., Deretz S., Le Roy P., Quillet E., Perez J.M. (Eds). INRA Prod. Anim., 24, 331-340.

Rousseau C., 2012. La coopération face à l'arrivée de la sélection génomique dans les schémas ovins laitiers français : une analyse stratégique des enjeux organisationnels. Ecole Nationale Supérieure des Sciences Agronomiques de Bordeaux, Bordeaux, p119.

Verrier E., Le Mezec P., Boichard D., Mattalia S., 2010. Evolution des objectifs et des méthodes de sélection des bovins laitiers. Bul. Acad. Vét. France, 163, 73-78.

Verrier E., Saint-Dizier M., 2011. Contraintes et opportunités pour l'amélioration génétique des ruminants. In : Numéro spécial, Amélioration génétique. Mulsant P., Bodin L., Coudurier B., Deretz S., Le Roy P., Quillet E., Perez J.M. (Eds). INRA Prod. Anim., 24, 297-306.

Vissac B., 2002. Les vaches de la République. Saisons et raisons d'un chercheur citoyen. 1-506.

\section{Résumé}

Après une présentation rapide du dispositif national historique de sélection des ruminants, cet article aborde les bouleversements politiques et techniques récents auquel celui-ci est confronté, notamment la sélection génomique, dans deux espèces : les bovins et les ovins laitiers. L'accent est mis sur l'analyse des changements organisationnels liés à cette nouvelle technique de sélection, dans quatre domaines des activités de sélection : $i$ ) les rapports entre acteurs de la recherche et acteurs de la sélection : développement de nouvelles formes de partenariats public-privé, de consortium, mais aussi développement de dynamiques privées de recherche, avec une évolution des relations historiques entre les opérateurs publics (INRA) et les entreprises; ii) les relations entre opérateurs de la sélection, avec un regroupement de ceux-ci, mais aussi une remise en question des relations de coopération historiques du fait de l'augmentation de la concurrence et des possibilités offertes par la génomique ; iii) les relations et les pratiques entre acteurs de la sélection et éleveurs, du fait de l'accélération de la production de données et iv) la gouvernance des races, du fait du développement de critères de sélection « privés » pouvant remettre en question la gestion en « bien commun » de la race. Une démarche d'accompagnement des dispositifs de sélection en ovins laitiers à la mise en œuvre de ces changements est présentée, montrant comment des acteurs s'organisent pour anticiper ces changements et maintenir les dispositifs collectifs. 


\begin{abstract}
Organizational implications of genomic selection in French dairy cattle and sheep: from diagnosis to a collective action

After a brief presentation of the historical national organization of breeding activities in France, this article considers the recent political and technical changes in the dairy cattle and sheep industry, more specifically genomic selection. It analyses the organizational implications of this new breeding technique in four areas of breeding activities: $i$ ) between research actors and breeding actors, with the development of new forms of public-private partnerships, and the development of private research initiatives, with evolving relations between public research and breeding companies; ii) among breeding actors, with increasing merging of companies and historical actors changing strategies from cooperation to competition; iii) between breeding actors and farmers, due to accelerating data production; $i v$ ) in the governance of the breed, due to the development of private breeding criteria questioning the management of the breed as a "common good". The article details an intervention-research process implemented to help sheep breeding actors face such changes, showing how they anticipate changes to maintain the collective organization of breeding activities and favor cooperation.
\end{abstract}

LABATUT J., ASTRUC J.-M., BARILLET F., BOICHARD D., DUCROCQ V., GRIFFON L., LAGRIFFOUL G., 2014. Implications organisationnelles de la sélection génomique chez les bovins et ovins laitiers en France : analyses et accompagnement. INRA Prod. Anim., 27, 303-316. 